\title{
ATOMIC P,HYSICS EXPERIMENTS WITH STORED COOLED HEAVY ION BEAMS
}

\author{
S. DATZ \\ Physics Division \\ Oak Ridge National Laboratory* \\ Oak Ridge, TN 37831-6377
}

\author{
CONF-861114--15 \\ DE87 001835
}

\section{Abstract}

The wide ranging interest in the development of heavy ion synchrotrons with electron beam cooling is evident from the number of projects presently under way. Although much of the initial motivation for these rings stemmed from nuclear and particle physics, a considerable amount of atomic physics experimentation is planned. This paper surveys some of the new opportunities in atomic physics which may be made available with storage ring systems.

\section{Introduction}

The primary motivation for the use of cooled heavy ion storage rings in atomic physics is the study of multiply charged ions. The intrinsic interest in the multiply charged ion physics from the possibility of using simple atomic systems, in which all the forces are known, to test concepts in quantum mechanics and quantum electrodynamics and to test solutions of the many-body problem. Multiply charged ions are particularly useful in these pursuits because the strong Coulomb field of the nucleus

\footnotetext{
${ }^{*}$ Research sponsored by the U.S. Department of Energy, Division of Chemical Sciences, Office of Basic Energy Sciences under Contract No. DE-ACO5$840 R 21400$ with Martin Marietta Energy Systems, Inc.
} 
acts to decoup,le electron-electron interactions and because there are large QED and relativistic effects on the energy levels of the system. The native habitat of multiply charged ions is in high temperature plasmas, such as those found in stellar atmospheres or fusion devices. Rapidly moving heavy particles, such as those created in a nuclear fission event, are multiply charged when they interact with matter. The energy levels of multicharged ions are widely spaced, leading to the emission of $X$-rays from relaxation of outer shell states of the $i o n$, and hence, the possibility of, for example, $X$-ray lasers from these systems. The need for information, concerning the structure and collision processes of multiply charged ions, in other branches of science and various developing technologies is manifold [1].

Unfortunately, many of the most interesting and important processes have not been available for study because of the limitations on the capabilities of experiments utilizing plasma sources and ion beams. With plasmas the motion is too random and the collision energy distribution too great, and with ion beams the currents are too low and the interaction times too short for a full and effective program of experimentation. If the ions, however, are stored in a synchrotron ring, they circulate in a closed loop and may pass the same point a million times a second, thereby greatly increasing the effective current.

The advantages of storage rings in creating significant new opportunities has been recognized by the physics community and, although these rings were initially conceived for use in nuclear physics, their applicability to atomic physics has become evident to a number of groups 
and three projects which to a large extent are atomic physics oriented are also under construction in Europe. These are at Stockholm's Research Institute for Physics, the University of Aarhus in Denmark, and the Max Planck Institute for Nuclear Physics in Heidelberg, W. Germany. All three are rings with a bending strength of about 1.5 tesla meters $(T \cdot m)$. The injector at Stockholm is a cryogenic electron beam ionization source (CRYEBIS) coupled to a radio frequency quadrupole (RFQ) linear accelerator; at Aarhus it is an isotope separator or an EN tandem Van de Graaff; and at Heidelberg it is the MP tandem-linac system. Larger rings which are dedicated primarily to nuclear and particle physics at Uppsala (Sweden), GSI (West Germany), CERN (Switzerland), and Tokyo (Japan) will also have atomic physics components in their programs. A list of these projects is given in table 1. Other snychrotron rings under construction at the University of Indiana and at Jülich, W. Germany (COSY) are, at the moment, exclusively dedicated to light ion nuclear physics. Subsequant talks in this symposium will cover programs from Heidelberg [2] and GSI [3], and descriptions of proposed projects at ORNL [4] and BNL [5]. Although each of these facilities has a unique set of capabilities, to the layman's eye the overall designs look remarkably similar. They comprise a four-to-six membered lattice with appropriately placed di-, quadru-, and hexapoles, an appropriate means of beam injection and (in some cases) beam extraction, an accelerating (and, in some cases, a decelerating) region, and an electron beam "cooling" region in which the circulating ion beam is inerged with a continuously replenished "cold" electron beam moving at the same velocity [6]. 
The storage time for multiply-charged ions will be controlled by charge transfer on background gas and hence on the beam velocity and vacuum attainable in the ring. The vacuum requirements for some sample cases in the Stockholm CRYRING system are given in table 2 [7]. Clearly $10^{-12}$ Torr vacua are strongly desired and a number of installations have already achieved this in test stand operation.

The attainable circulating currents depend on the mode of injection, the energy of injection and circulating beam energy. Some examples of anticipated currents for eight turn injection into the ORNL HISTRAP facility are shown in table 3. Although considerable advantage is obtained with higher energy injection, the reader should note that the currents listed are in units of milliamperes.

Several workshops have been held to jnvestigate the opportunities offered by heavy ion storage rings $[8-10]$ and a study of the subject by the NAS/NRC Committee in Atomic and Molecular Sciences is presently underway. The function of the present paper is to outline the possibilities presented by Ring Atomic Physics.

\section{Electron-Ion Interactions}

The dominant processes which occur in high temperature plasmas are controlled by electron-ion collisions. 'These give rise to excitation, ionization, and recombination (radiative and dielectronic) which, in turn, control the ionic charge-state distribution and the composition and flux of radiation emitted from the plasmas.

The electron beam "cooling section" of the storage ring offers an almost ideal system for the study of these processes. In this region a 
space-charge-limited electron beam will be merged with the stored ion beam, permitting the study of low relative energy electron-ion collisions at high laboratory energies. The advantages of working with high laboratory velocities and a ring configuration can be seen from the following considerations.

To achieve the desired $v_{r}$, a high $v_{i}$ requires a high electron velocity, $v_{e}$, and for space-charge-limited beams $\rho(e) \propto v^{3 / 2} / v_{e} \propto v_{e}{ }^{2}$. The rate $R$ at which a process with cross section $\sigma$ occurs in the merged beam is $R=\sigma\left(v_{r} / v_{j}\right) \rho(e) 2 \phi_{j}$, where $v_{r}$ is the relative velocity, $v_{j}$ is the ion velocity, $\rho(e)$ is the electron density, $\phi_{j}$ is the ion current, and $\ell$ is the length of the overlapping beam region. For a given $\triangle P / P$ value, the ion current in the circulating beam at the Keil-Schnell stability limit $\phi_{j} \propto v_{j}{ }^{3} / L$, where $L$ is the ring's circumference. Since in most experiments $v_{\varrho} \simeq v_{i}$, we have

$$
R \propto V_{j}{ }^{4}(\ell / L) \text {. }
$$

(Note that the ion space-charge limit which one can load into the ring depends on the injection energy, hence the desire in this case for high energy injection.)

\subsection{Recombination}

Two processes are known to be important in the recombination of ions with free electrons: radiative recombination $(R R)$ in which a photon is released whose energy is exactly equal to the potential energy gain and dielectronic recombination (DR) in which a continuum electron excites a 
previously bound electron and in so doing loses just enough energy to be captured into a bound state $(n \ell)$. The latter process results in a doubly excited ion in a lower charge state which may either autoionize or emit a photon resulting in a stabilized recombination. In both cases the result is an ion of reduced charge and a photon. The principal background in these experiments arises from electron capture from residual gas. Since capture cross sections decrease very strongly with $v_{j}\left(\propto v_{j}^{-1}\right)$, the advantage of high $v_{j}$ (in the laboratory system) is again very evident.

\subsection{Radiative recombination}

Radiative recombination (RR) peaks at $v_{r}=0$, and it is, in fact, a process which competes with electron beam cooling. The magnitude and energy of dependence of the cross section for RR into a given $n$ state of bare ions are well-known theoretically,

$$
\sigma_{R R}(n)=2.11 \times 10^{-22} \frac{E_{0}{ }^{2}}{n E_{\ell}\left(E_{0}+n^{2} E_{\ell}\right)} \mathrm{cm}^{2} \text {, }
$$

where $E_{0}$ is the ionization potential and $E_{\ell}$ is the electron energy. The result of the recombination is an ion of reduced charge and the emission of a photion. Measurement of the number of reduced charge ions as a function of electron beam energy can be used to diagnose the electron beam cooler region.

Because of the high luminosity of the stored beams, it should also be possible to measure light emitted from the merged beam region. Measurement of the photon energy for RR to an initially bare ion can give direct information on the 15 binding energy and the 1s Lamb shift. 
Although, RR for bare ions is well understood, RR cross sections for capture into low $n$ states of ions with already bound electrons are not. This is a problem of some considerable interest in high temperature plasmas. Here studies of the RR emission spectra will yield valuable information on the $n$ dependence of RR cross sections for these systems.

\subsection{Dielectronic recombination}

Unlike radiative recombination in which the dependence of the cross section on energy is continuous and monotonic (1/E), dielectronic recombination is characterized by a set of resonances. The process may be represented as follows: an ion $A$ of charge $q+$ in its ground state $\alpha$ is excited to a core state $\beta$ and captures the electron into a given $(n, l)$ state,

$$
A q+(\alpha)+e \neq[A(q-1)+(\beta, n \ell)]^{* *} \rightarrow A(q-1)+(\alpha, n \ell)+h \nu .
$$

The doubly-excited intermediate decays either by autoionization to its original pair or radiatively into a recombined ion. Because the recombination here requires an excitation of the ion, higher collision energies (temperatures) are generally required.

The only reported measurements of DR on multiply-charged ions have been those of the ORNL group. There muiltipiy charged ion beams obtained from an EN Tandem Van de Graaff were merged with a compressed space-chargelimited electron beam. The recombination cross sections were obtained from the fraction of reduced-charge ions as a function of relative energy. 
On the positive side, results have been obtained for Li-like, B-like, Be-like, and Na-like ions up to $\mathrm{Cl}^{6+}$. On the negative side are first, $\Delta n>0$ transitions have not been observable because of their lower cross section and second, the fact that tine energy resolution in these experiments $(\sim 5 \mathrm{eV})$ is insufficient to resolve individual resonances.

The ORNL experiment is particularly germane here since it resembles in concept a single pass of the ion beam through the "cooler" region and by contrast points up the advantage of the ring system. With a resolution of 0.1-0.2 ev ring experiments would be able to resolve structure and individual resonances in the $D R$ spectrum. The high luminosity offered by the ring would allow measurements of cross sections which are orders of magnitude lower than those obtained in the single-pass experiment. Backgrounds in the single-pass experiments arise from charge capture from residual gas and from slit edge scattering: these effects are much mitigated in the ring because of the higher ion beam velocity, better background pressure, and the complete absence of slits in the system.

The recent experiments have shown significant flaws in the previously accepted theory, and the present interaction between theory and experiment has been highly iterative. All of this has pointed up the need for further and more detailed information on systems over a wide range of charge states.

\subsection{Laser-induced radiative recombination}

As we have seen above, the radiative recombination cross section $\sigma_{R R}(n) \propto q^{2} /$ ne. By merging a laser beam with the merged electron-ion beam, 
we should be able to populate preferentially a given $n l$ state in a recombined $(q-1)+$ ion $[12]$. If the $(q-1)+$ ion fits into the ring's momentum aperture, we should be able to store and build up population in selected states [13]. The ratio $g(n)$ between the rate for spontaneous recombination to induced recombination is given by

$$
g(n)=u(v)(B / A),
$$

where $u(v)$ is the spectral energy density and $A$ and $B$ are the Einstein coefficients for spontaneous and induced recombination, respectively, with $A=8 \pi\left(h v^{3} / C^{3}\right) B$. With available laser powers in the visible and UV regions, enhancements by factors of 100 to 500 should be possible. For very highly stripped $i$ ons, photons in the $2-$ to $5-e V$ range would enhance Rydberg levels. By choosing high $\ell$ values, one might be able to store long-lived states of the $(q-1)+$ ions for separate experimentation.

\subsection{Laser-induced dielectronic recombination}

Dielectronic recombination can be thought of as a two-step process. The first involves the formation of a doubly excited state of the recombined icr $[A(q-1)+(\beta, n l)]^{\star \star}$ and the second as a radiative stabilization which drops the total energy of the ion below tlic autoionizing ?imit. The cross section for dielectronic recombination is proportional to

$$
\sigma_{D R} \propto \frac{A_{A} \Sigma A_{R_{i}}}{\sum\left(A_{R_{j}}+A_{A_{j}}\right)},
$$

where $A_{A}$ is the autoionizing rate of the initial state and $A_{R_{j}}$ and $A_{A_{j}}$ are the radiative and autoionizing rates for any of $i$ pathways for 
relaxing the intermediate state. Recombination to a specific $n \ell$ state could be laser enhancea by increasing the radiative rate for either the $\beta \rightarrow \alpha$ relaxation or a $(n \ell) \rightarrow\left(n^{\prime} \ell^{\prime}\right)$ transition. The $\beta \rightarrow \alpha$ transition energy will be a function of the nl state of the second electron; hence, the enhanced recombination rate will be tuneable to a given $(n, l)$ value. In most cases, presently available laser photons are too low in energy to induce the $\beta \rightarrow \alpha$ transition. However, some cases of $(n l) \rightarrow\left(n^{\prime} \ell^{\prime}\right)$ pumping appears feasible.

\subsection{Ionization and excitation}

Experiments on ionization and excitation are presently carried out using crossed beams of electrons with multicharged ions obtained from ECR or EBIS sources. For multicharyed ions these experiments have yielded a number of surprises $[14,15]$. A simple universal formula due to Lotts pre. dicts that the ionization cross section should have a gradual slope at threshold, rise to a broad peak, and then decline as $(\log E) / E$, where $E$ is the collision energy. For a number of multicharged ions, this formula underestimates the ionization cross section by more than an order of magnitude! Moreover, detailed structure is visible in the energy dependence. This enhanced cross section and structure is due to the formation of autoionizing states by excitation of inner shell electrons. The limitations of present techniques are twofold. First, the smali cross sections require intense crossed electron beam currents which, in turn, leads to poor energy resolution and hence inability to resolve details of the structure. Second, the lower limit on the size of the cross 
sections that ,can be measured using crossed beams precludes measurement of high ionization stages.

The availability of cooled heavy ion storage rings could do much to remove these limitations and open some new areas of investigation. The experiments would be carried out in the merged electron beam region, and the signal would be in the form of ions of higher charge $(q+1)+$. The advantages here are the increase in signal because of the much higher luminosity, and the greatly increased energy resolution because of the merged-beam kinematics and the nature of the "cooling beam." Because of the increased energy resolution, sharp thresholds in the energy dependence due to inner shell excitation should become visible, and the measurement of positions of these discontinuities can be used in atomic structure determination.

Because of the recycling through the electron beam, it may be possible to measure outer shell excitation by multielectron ionization. If the relative energy $E_{r}$ in the electron-ion merged-beam region is scanned, $(I / 2)<E_{r}<I$, where $I$ is the ionization potential. Direct ionization cannot occur, but excitation to some level in this region could. If this level is either metastable or cascades to a metastable state with lifetimes long compared to $\sim 10^{-6} \mathrm{sec}$ (the ring transit time), the ion may be ionized by a second electron collision while passing through the merged-beam region again. This would be evidenced by ionization events occurring for $E_{r}<I$. Since we would really be measuring excitation, the thresholds in cross section should be sharp rather than gradual. 
An interesting and important additional point here is that metastable states of ions injected into the ring can be removed by simply allowing them to relax during storage. These ions can perturb considerably measurement of any collision cross section (e.g., Be-like ions for $z=5-10$ atoms are generally formed with about $70 \%$ in the metastable $1 s^{2} 2 s 2 p^{3} p$ states [16] which have lifetimes $>10^{-6} \mathrm{sec}$ ).

\subsection{Condensed "crystalline" beams - the cooliny process}

The "cooling process," i.e., the process by which the emittance and momentum spread of the beam is reduced, is in itself an interesting area for fundamental investigation. The "cooling" process proceeds through Coulomb scattering of cold electrons from "hot" ions. To see this in a simple manner, imagine a valume filled with perfectly stationary electrons (i.e., assume $\mathrm{T}_{e}=0^{\circ} \mathrm{K}$ ) which is moving in the laboratory system with a velocity exactly equal to the average velocity of a group of positively charged ions. Ions with velocity components exactly equal to the electron group velocity will be unaffected, but ions with any remaining velocity in any direction will be slowed by Coulomb scattering. In the ring the ion beam passes through a freshly supplied cold electron beam region on each pass and should ultimately reach the electron "temperature."

The actual temperature distribution of a beam of electrons accelerated from a cathode at temperature $T_{C}$ is "flattened" in space [17]. The transverse temperature of the beam $T_{\perp}$ is equal to $T_{C}\left(i . e, k T_{C}=0.1-0.2 \mathrm{eV}\right)$, but because the beam is accelerated to an energy $E$, the longitudinal temperature $T_{\|}$is decreased to 


$$
k T_{\|}=1 / 8\left(k T_{C}\right)^{2} / E \text {, }
$$

so that, for example, for a $1-\mathrm{keV}$ electron beam $T_{\|} \sim 10^{-20} \mathrm{~K}$.

If such cooling can actually be achieved (evidence for its occurrence already exists from some experiments at Novosibirsk), it is possible that a "crystallization" of the ion beam could occur [18]. In the reference frame of the ion beam center, an individual beam particle rotates in a small circle (i.e., $\sim 100-\mu m$ dia.) because of the strong focussing transverse betatron oscillation. To minimize their potential energy, the ions would order themselves with a fixed interionic distance in the longitudinal dimension. One might be able to detect such a structure by permitting the beam to escape the ring and be swept electrostatically across a position-sensitive detector. With a linesrly increasing deflection voltage the ordered structure in the beam should turn up as equally spaced spots on the position-sensitive detector.

If such a crystallized beam can, in fact, be formed, many interesting properties rollow. On the practical side, for example, only adiabatic collisions between beam particles can orcur because of the order in the crystal. Intrabeam scattering is eliminated for the same reason. Questions relating to this new solid's structure include its lattice "phonon" spectrum and the coherence lengths in lattice oscillations.

\section{Ion-Atom Collisions}

The discussions above have dealt with processes in which the ion beam was confined to the ring and the interacting species did not disturb the 
vacuum. For experiments involving atomic targets, we must be able to either to introduce gas into the ring in the form of a differentially pumped cross beam or to extract the stored beam. Moreover, to obtain the appropriate center-of-mass anergies, we must either be able to vary the ion beam energy or be able to merge an accelerated atom (neutralized ion) beam.

The advantages obtained in cooled storage ring. systems are again those of emittance and luminosity. Beams of very high charge state ions available from tandem accelerator injection can be decelerated and cooled in the ring for low-energy collisions studies.

The high luminosity permits use of very thin targets to achieve reasonable signal levels for low cross section processes in the presence of, e.g., sequential high cross section processes which lead to the same product. An example here is the study of electron pair transfer from He to an energetic highly charged ion. The cross section for singleelectron transfer may be $10^{3}$ to $10^{4}$ times greater. To avoid the interference of sequential single capture would require a vanisningiy small target length for a single-pass experiment. However, with a target of just $10^{10}$ atom $/ \mathrm{cm}^{2}$ and a cross section of $10^{-22} \mathrm{~cm}^{2}$, the $1-\mathrm{mA}$ beams in the storage ring would give signals of $\sim 6000 \mathrm{cps}$ with insignificant interference from double capture.

Such intensities also make possible measurements of the emission spectra following charge capture into excited states of the circulating ion. By colliding a bare nucleus with a spin polarized atomic target, such as an optically pumped lithium beam, one may prepare and store a 
spin-polarized, hydrogenlike ion beam for future experiments. Here is another example of the utility of the multicharge storage capability. In connection with charge transfer of polarized electrons, it also has been suggested that parity violation might be observed in an experiment where one measures the polarization of the radiative electron capture photon in coincidence with the electron spin direction [19].

\subsection{Generation of very low-energy multicharged ion beams}

It has been demonstrated that fast (MeV/nucleon) multicharged ions colliding with neutral target atoms can create multicharged recoiling target ions with high probability. These recoiling target ions have very little kinetic energy ( $\leqslant 1 \mathrm{eV})$ and can be used for further experimentation. The recoils are generally extracted from the collision region, charge and energy analyzed, and used in farther collision studies in the keV energy region [20]. Thus far, "hammer beams" (i.e., the MeV/nucleon beams) have been supplied by many accelerators, ranging from EN-Tandem Van de Graaffs to the UNILAC at GSI. The advantage of the HISTRAP for such experimentation lies in the intense currents of the stored ions available for use as "hammers." If the cross section for multiple target ionization is much greater than the charge-changing cross section of the projectile ion, the individual projectile ion may be recjcled in the ring to create many target recoils. (The energy loss in these collisions may be recovered by the electron beam "cooler.") If one has multicharge storage capability, charge-changing collisions of the "haminer" ion will not destroy its utility as long as its charge state, $q$, can be accommodated in the ring. Therefore, 
if one injects, the ion into the ring at its most probable equilibrium charge value, it will be useful as a "harmer" until it leaks out through collisions which take it outside an acceptable $\Delta q$ window.

Estimates by the Frankfurt group [21] indicate that intensities of $10^{9}$ bare recoil Ar ions per second would be produced from a $10^{11}$ atom $/ \mathrm{cm}^{2}$ target by $10^{10}$ iodine ions circulating at $8 \mathrm{MeV} /$ nucleon. Such intensities of very low-energy $\mathrm{Ar}^{18+}$ have never been approached. in the past and would open new possibilities for experimentation (e.g. differential scattering, crossed beams with the ECR multicharged ion beams, etc.).

\subsection{Merged-beam ion-icn collision experiments}

The availability of two separate injection systems such as will be found on HISTRAP makes possible an interesting and unique opportunity for the study of ion-ion collisions. Here, one would inject an ion beam with a given $M / q$, where $M$ is the mass of the ion having charge $A$, from the ECR-RFQ injector, accelerate it to a kinctic energy $E$, and then inject another ion species from the Holifield tandem. The condition for the simultaneous storage of two ion beams is (nonrelativistically)

$$
\left(E_{1} / E_{2}\right)=\left(M_{2} / M_{1}\right)\left(q_{1} / q_{2}\right)^{2}
$$

Within the merged beams, collisions would take place with relative energy

$$
E_{r}=k \mu(B \rho)^{2}\left[\left(q_{1} / m_{1}\right)-\left(q_{2} / m_{2}\right)\right]^{2},
$$

where $\mu$ is the reduced mass and $B P$ the magnetic rigidity of the system. A continuous range of $E_{r}$ is obtained by varying $E_{1}$ and suitably adjusting $E_{2}$ 
and $B$. For experiments of this type, such as those involving colliding beams, good emittance is necessary. Although the two beams are at different velocities, cooling can be achieved by changing the electron beam velocity to accommodate each beam separately. Studies of charge transfer, ionization, and other collisional phenomena in ion-ion systems have been very strongly circumscribed in the past, and the capabilities of some future ring systems may do much to open this area to investigation.

\section{Spectroscopy}

\subsection{Precision spectroscopy of one- and two-electron ions}

The objective of work in this area is to achieve rigorous tests of single-particle quantum electrodynamics over a wide range of nuclear charge and to study specific two-electron correlations and level shifts [22]. Experiments at various accelerators arourd the world have used beam foil spectroscopy, low energy recoil ions, and deceleráced ion beams for these measurements. Problems of Doppler width and "spectator" electrons (i,e., a second electron captured into some outer orbit which perturbs the inner energy levels) have limited the accuracy of the measurements. At the moment, using presently available methods, the decelerated totally stripped ion beam is the most promising. But even here the potentialities are limited by Liouville's theorem since the deceleration leads to an increased angular width which mitigates the Doppler width gain from deceleration.

Cooled stored beams offer great advantages here. The precise energies of the beam are known, and the emittance can be very highly refined. The ions can be decelerated and the attendant increase in angular width can be removed by electron beam cooling. 
To perfor,m such experiments, a beam of bare nuciei is formed, for example. The nuclei are allowed to capture an electron into in excited state from some target. The precision spectroscopy is then done on the radiative decay photons. For the highly charged ions in question, these photons are in the $X$-ray region.

A ring experiment could be carried out either with a decelerated cooled extracted beam passing through a thin gas target or with the stored beam at higher velocities. It is estimated that a precision in Lamb shift measurements of $10^{-4}$ may be possible using stored cooled beams [22].

\subsection{High resolution laser spectroscopy of ions}

Storage rings could supply an excellent source for high resolution linear and nonlinear laser spectroscopic studies of ions. One arm of the ring could be used as a common path for collinear laser and particle beams. The laser beam could provide either a traveling wave field (for linear spectroscopy) or a standing wave field (for nonlinear spectroscopy, such as saturation or two-photon absorption spectroscopy).

The finite line widths of spectral lines limit the ultimate precision attainable in high resolution measurements. The major cause of line broadening for atoms or ions in rare-field media is the first-order Doppler effect. The compression of the longitudinal velocity distribution of the ions in the ring, resulting from kinematic bunching and electron cooling, could reduce considerably the size of this contribution in a collinear laser-particle beam arrangement. The first-order Doppler effect could be eliminated entirely by using, for example, two-photon absorption spectroscopy. 
If first-order Doppler broadening is eliminated or sufficientiy reduced, the residual line width will be determined primarily by the finite time available for the spectroscopic measurement. In effect, this time is the duration of the coherent interaction between a particle and the electromagnetic field. This time interyal may be determined in a given experiment by either the traisit times of particles through the interaction region, the duration of the optical field if the laser is operated in the pulsed mode, or the radiative lifetime of the upper and lower levels of the transition. In a single interaction region the line width will be determined by the shortest of these times. However, the restriction set by the uncertainty principle can be overcoine by the use of a quantum interference method based on the interaction of the particle with two or more temporally separated interaction regions. In the case of the ring one could visualize the particles interacting with a collinear laser field situated in one arin of the ring on every orbit of the ring. In principle, this should narrow the resonances considerably. The arrangement would produce results like the multiple-slit interference pattern obtained with a diffraction grating. of course, it is essential that the phase difference between successive interaction regions remains fixed, i.e., coherent.

Many single-charged ions have E1 transitions which may be pumped by presently available lasers. In multicharged ions, with the important exception of Rydberg states, most El transitions have too high an energy to be pumped. However, there are a number of Ml transitions between hyperfine states available in this frequency range which, with presently available laser intensities, could be used in the ring experiments [23]. If the 
intensity and,resolution available from synchrotrons is sufficient a much higher frequency range can be made available [24].

\subsection{Molecular ion spectroscopy}

Studies of the structure of simple molecular ions is a rapidly growing field. Presently, the ions are studied in gas discharges or by Doppler turing an ion beam velocity to a laser frequency in a merged-beam system. In a sense this is similar to the single-pass merged electron-ion beam experiments described in the section on recombination. Here again the luminosity and immersion time available in a ring system may do much to increase the sensitivity and precision of the experiments. In the ring system the molecular ionic target would be exposed many times to the stimulating photon field during the lifetime of an excited state. In effect the merging of the ion beam with a photon' beam in one leg of the ring is equivalent to a Ramsey separated resonator experiment.

An additional benefit which may be obtained in a storage ring is the ability to allow time for the radiative decay of internai excitation (IR active inodes) which may have been introduced in the ion source.

\subsection{Laser "cooling" of ion beams}

If a laser beam directed counter to an ion beam is tuned for resonant absorbtion by the ion, the ion will be decelerated. This occurs because the absorbtion of the directional momenta of the incident photons is followed by an isotropic distribution of the re-emitted photons. If there is a distribution of velocities in the ion beam, the deceleration will only take place for those ions which are not Doppler shifted out of 
resonance with the laser beam. Thus, for example, by tuning the laser (or ion beam velocity) so that resonance is achieved for the higher velocity particles, the distribution can be squeezed towards the lower velocity edge and cooling is achieved. With singly charged ions, where dipole allowed transitions (E1) are accessible with ordinary lasers, cooling times are of the order of $10 \mathrm{msec}$ [25]. For multicharged ions El transitions require photons which are too high in energy for presentiy available lasers. However, transitions between, $€ . g .$, Ml transitions in intercombination states of highly charged ions, do fall into the visible region. Although oscillator strengths may be $10^{4}$ lower than for El transitions, saturation is still achievable. The limitation here is in the relaxation time of the excited state which may exceed the transit time around the ring. If this were the case, suitable laser beam modulation would have to be eraployed. The interplay between Doppler shift and the precision with which a given intercombination line is known wolla permit, on the one hand, a precise calibration of the beam energy or, on the other, precise spectroscopy of the ionic system, 
References ,

[1] S. M. Younger, Nucl. Instrum. and Methods (these proceedings).

[2] R. Schuch, Nucl. Instrum. and Methods (these proceedings).

[3] B. Franzke, ilucl. Instrum. and Methods (these proceedings).

[4] R. F. 01sen, Nucl. Instrum. and Methods (these proceedings).

[5] K. W. Jones, Nucl. Instrum. and Methods (these proceedings).

[6] F. E. Mills, Nucl. Instrum. and Methods (these proceedings).

[7] C. J. Herrlander, L. Bagge, A. Barany, S. Borg, H. Danared, P. Heikkinen, S. Hultberg, L. Liljeby, and Th. Lindblad, IEEE Trans. Nucl. Sci. NS-32, No. 5 (1985) 2718.

[8] Workshop on the Physics with Heavy Ion Cooler Rings (Heidelberg 1984).

[9] Proceedings "ECO0L '84" (H. Poth, ed.), KfK Karlsruhe (1984).

[10] Proceedings, Workshop on Atomic Physics with Stored Cooled Heavy Ion Beăms (S. Datz, ed.), DOE-CONF-860144 (1986).

[11] P. F. Dittner, S. Datz, P. D. Miller, C. D. Moak, P. H. Stelson, C. Botticher, W. B. Dress, G. D. Alton, N. Nesković, and C. M. Fou, Phys. Rey. Lett. 51 (1983) 31; P. F. Dittner, S. Datz, P. D. Miller, P. L. Pepmiller, and C. M. Fou, Phys. Rev. A33 (1986) 124; S. Datz, P. F. Jittner, C. M. Fou, P. D. Miller, and P. L. Pepmiller, Nucl. Instrum. and Methods, Proceedingsi of Int'1. Confereace on the Physics of Multiply Charged Ions, in press.

[12] R. Neumann, H. Poth, A. Winnacker, and A. Wolf, Z. Physik A313 (1983) 253.

[13] This feature is planned for the Heidelberg and ORNL rings. 
[14] D. C. Gniffin, C. Bottcher, M. Pindzola, S. M. Younger, D. C. Gregory, and D. H. Crandall, Phys. Rev. A29 (1984) 1729.

[15] D. C. Gregory, Nucl. Instrum. Meth. B10/11 (1985) 87.

[16] S. Datz, P. F. Dittner, H. F. Krause, P. D. Miller, P. L. Pepmiller, Y. Yamazaki, and N. Stolterfoht, Bull. Am. Phys. Soc. 31 (1986) 974.

[1?] Ya.S. Debrenev and A. N. Skrinsky, Particle Accelerators $\underline{8}$ (1988) 1;

A. H. Sorensen and E. Bonderup, Nucl. Instrum: Meth. $\underline{215}$ (1983) 27; and A. H. Sorensen in ref. [9].

[18] N. S. Dikansky and D. V. Pestrikov in ref. [9], p. 275;

J. P. Schiffer and P. Kienle, 2. Physik A321 (1985) 181; and J. P. Schiffer and 0. Poulsen, preprint (1985).

[19] F. Bosch, paper D-11 in ref. [8].

[20] See, e.g., C. L. Cocke, J. P. Giese, L. N. Tunne11, W. Waggoner, and S. L. Varghese in "Electronic and Atomic Collisions," D. C. Lorentz, W. E. Meyerhof $f_{2}$ and J. R. Peterson, eds., Elsevier Science Publishers (1986) pp. 453-461.

[21] H. Schmidt-Böcking in ref. [8].

[22] R. D. Deslattes in ref. [10].

[23] T. Kueht and G. Huber in ref. [8].

[24] B. M. Johnson in ref. [10].

[25] J. Javanainen, M. Kaivola, U. Nielsen, O. Paulsen, and E. Riis, J. Opt. Soc. Am. B2 (1985) 1768. 


\section{Tabie 1. Heavy Ion Storage Rings with Electron Beam Cooling Presently under Construction}

\begin{tabular}{|c|c|c|}
\hline Site & $\begin{array}{l}\text { Approximate } \\
\text { Strength }\end{array}$ & $\begin{array}{l}\text { Heavy Ion } \\
\text { Injection }\end{array}$ \\
\hline Aarhus, Denmark "ASTRID" & $1.8 \mathrm{~T} \cdot \mathrm{m}$ & $\begin{array}{l}200 \mathrm{keV} \text { isotope } \\
\text { separator or EN } \\
\text { tandem }\end{array}$ \\
\hline Heidelberg, W. Germany "TSR" & $1.5 \mathrm{~T} \cdot \mathrm{m}$ & MP Tandem + Linac \\
\hline Stockholm, Sweden "CRYRING" & $1.4 \mathrm{~T} \cdot \mathrm{m}$ & CRYEBIS + RFQ \\
\hline CERN, Switzerlanda) "LEAR" & $7 \quad \mathrm{~T} \cdot \mathrm{m}$ & Linac - RFQ \\
\hline GSI, Darmstadt, W. Germany "ESR" & $10 \quad T \cdot m$ & GSI - UNILAC \\
\hline Uppsala, Swedenb) "CELCIUS" & $7, T \cdot m$ & Cyclotron \\
\hline Tokyo, Japanb! "TARN II" & $6.9 \mathrm{~T} \cdot \mathrm{m}$ & Cyclotron \\
\hline
\end{tabular}

a) Electron team cooling and heavy ion capability to be added to this functioning facility.

b) Heavy ion capability is planned for future. 
Table 2. Vacuum requirements in CRYRING under different. operational conditions

\begin{tabular}{c|cc}
\hline Ion & \multicolumn{2}{|c}{ Torr } \\
\cline { 2 - 3 } & $2 \times 10^{-10}$ & $2 \times 10^{-12}$ \\
\hline $\mathrm{Ar}^{18+}$ & $5 \times 10^{-11}$ & $1 \times 10^{-12}$ \\
$\mathrm{Kr}^{34+}$ & $2 \times 10^{-11}$ & $7 \times 10^{-13}$ \\
$\mathrm{Xe}^{44+}$ & $7 \times 10^{-12}$ & $5 \times 10^{-13}$ \\
$\mathrm{Pt}^{64+}$ & & $\mathrm{b}$ \\
\hline
\end{tabular}

a. Storage mode, $E \mathrm{MeV} /$ nucleon, $T_{1 / 2}=60 \mathrm{~min}$.

b. Storage mode, $0.1 \mathrm{MeV} /$ nucleon, $\mathrm{T}_{1 / 2}=3 \mathrm{sec}$. 
Table 3

Injection $\quad 2 \mathrm{~T} \cdot \mathrm{m} \quad 0.1 \mathrm{~T} \cdot \mathrm{m} \quad 20 \mathrm{keV} /$ nucleon

\begin{tabular}{|c|c|c|c|c|}
\hline $\begin{array}{l}Q=6, A=12 \\
E / A(M e V) \\
\text { current }(m A)\end{array}$ & $\begin{array}{l}4.0 \\
1.69\end{array}$ & $\begin{array}{c}47.1 \\
5.61\end{array}$ & $\begin{array}{l}.121 \\
0.30\end{array}$ & $\begin{array}{l}.020 \\
0.12\end{array}$ \\
\hline $\begin{array}{l}Q=18, A-40 \\
E / A(M e V) \\
\text { current }(m A)\end{array}$ & $\begin{array}{l}6.6 \\
1.34\end{array}$ & $\begin{array}{c}38.3 \\
3.15\end{array}$ & $\begin{array}{l}0.10 \\
.164\end{array}$ & $\begin{array}{r}0.020 \\
.075\end{array}$ \\
\hline $\begin{array}{l}Q=43, A=238 \\
E / A \text { (MeV) } \\
\text { current (mA) }\end{array}$ & $\begin{array}{c}1.57 \\
.658\end{array}$ & $\begin{array}{l}6.28 \\
1.31\end{array}$ & $\begin{array}{l}.016 \\
.066\end{array}$ & $\begin{array}{l}.020 \\
.075\end{array}$ \\
\hline $\begin{array}{l}Q=5, A=12 \\
E / A(M e V) \\
\text { current (mA) }\end{array}$ & $\begin{array}{l}.300 \\
.0135\end{array}$ & $\begin{array}{l}32.9^{\prime} \\
1.38\end{array}$ & $\begin{array}{l}.083 \\
.00712\end{array}$ & $\begin{array}{l}.020 \\
.00352\end{array}$ \\
\hline $\begin{array}{l}Q=i 1, A=40 \\
E / A \text { (MeV) } \\
\text { current (mA) }\end{array}$ & $\begin{array}{l}.300 \\
.0297\end{array}$ & $\begin{array}{c}14.5 \\
.204\end{array}$ & $\begin{array}{l}.0364 \\
.0103\end{array}$ & $\begin{array}{l}.020 \\
.0078\end{array}$ \\
\hline $\begin{array}{l}Q=21, A=238 \\
E / A(M e V) \\
\text { current (mA) }\end{array}$ & $\begin{array}{l}.300 \\
.0056\end{array}$ & $\begin{array}{l}1.5 \\
.127\end{array}$ & $\begin{array}{l}.0036 \\
.0006\end{array}$ & $\begin{array}{l}.020 \\
.0015\end{array}$ \\
\hline
\end{tabular}

\section{DISCLAIMER}

This report was prepared as an accuunt of work sponsored by an agency of the I/nited States Government. Neither the United States Government nor any agency thereof, nor any of their employees, makes any warranty, express or implied, oi assumes any legal liability or responsibility for the accuracy, completenss, or usefulness of any information, apparatus, product, or process disciosed, or represents that its use would not infringe privately owned rights. Reference herein to any specific commercial product, process, or service by trade name, trademark, manufacturer, or otherwise does not necessarily constitute or imply its endorsement, recommendation, or favoring by the United States Government or any agency thereof. The views and opinions of authors expressed herein do not necessarily state or reflect those of the United States Government or any agency thereof. 